\title{
GENERAL PRINCIPLES OF LEARNING-BASED MULTI-AGENT SYSTEMS
}

\author{
David H. Wolpert \\ NASA Ames Research Center \\ Moffett Field, CA 94035 \\ dhw@ptolemy.arc.nasa.gov
}

\author{
Kevin R. Wheeler \\ NASA Ames Research Center \\ Caelum Research \\ Moffett Field, CA 94035 \\ kwheeler@mail.arc.nasa.gov
}

\author{
Kagan Tumer \\ NASA Ames Research Center \\ Caelum Research \\ Moffett Field, CA 94035 \\ kagan@ptolemy.arc.nasa.gov
}

\begin{abstract}
We consider the problem of how to design large decentralized multiagent systems (MAS's) in an automated fashion, with little or no hand-tuning. Our approach has each agent run a reinforcement learning algorithm. This converts the problem into one of how to automatically set/update the reward functions for each of the agents so that the global goal is achieved. In particular we do not want the agents to "work at cross-purposes" as far as the global goal is concerned. We use the term artificial COllective INtelligence (COIN) to refer to systems that embody solutions to this problem. In this paper we present a summary of a mathematical framework for COINs. We then investigate the real-world applicability of the core concepts of that framework via two computer experiments: we show that our COINs perform near optimally in a difficult variant of Arthur's bar problem [1] (and in particular avoid the tragedy of the commons for that problem), and we also illustrate optimal performance for our COINs in the leader-follower problem.
\end{abstract}

\section{INTRODUCTION}

In this paper we are interested in computational problems having the following characteristics:

- the agents each run reinforcement learning (RL) algorithms;

- there is little to no centralized communication or control;

- there is a provided world utility function that rates the possible histories of the full system.

There are many examples of such problems, some of the more prominent being:

i. Designing a control system for constellations of communication satellites or of constellations of planetary exploration vehicles (world utility in the latter case being some measure of quality of scientific data collected);

ii. Designing a control system for routing over a communication network (world utility being some aggregate quality of service measure);

iii. Construction of parallel algorithms for solving numerical optimization problems (the optimization problem itself constituting the world utility);

Appears in the Proceedings of the Third International Conference on Autonomous Agents, pp 77-83, 1999. iv. Control of a large, distributed chemical plant.

These kinds of problems may well be most readily addressed by using a large Multi-Agent System (MAS) [19], where each agent is restricted to communicate with only a few neighbors, and where each agent runs a Reinforcement Learning (RL) algorithm. In such systems, a crucial problem is ensuring that the agents' RL algorithms do not "work at cross-purposes", so that their collective behavior maximizes a provided global utility function. The difficulty in achieving this is that these systems have no centralized control, so the dynamics is governed by the collective effects of the individual agents each modifying their behavior via their (local) RL algorithms.

We are interested in such systems where the agents are "greedy" (i.e., there is no external structure forcing cooperation). However, both the agents reward functions and the overall system structure are automatically set and then updated in a machine learning-like fashion, so as to facilitate the achievement of the global objective.

As opposed to hand-tailored MAS design, these alternative approaches potentially have the following benefits: one does not have to laboriously model the entire system; global performance is "robust"; one can scale up to very large systems; and one can maximally exploit the power of machine learning. We use the term COllective INtelligence (COIN) [21, 22, 23] to refer to either MAS's designed in this way, or (in the case of naturally occurring MAS's) to MAS's investigated from this perspective.

The COIN framework is related to many other fields. (See [21] for a detailed discussion of these relations, involving several hundred references.) Some of them are:

- multi-agent systems;

- computational economics;

- reinforcement learning for adaptive control;

- statistical mechanics;

- computational ecologies;

- game theory, in particular, evolutionary game theory.

Previous MAS's most similar to a COIN include those where agents use reinforcement learning [8, 13], and/or where agents actively attempt to model the behavior of other agents [14].

In this paper we introduce some of the concepts from the COIN framework, and then present experiments testing those concepts. The restricted version of the framework presented here is not sufficient to formally guarantee optimal global performance for all multi-agent systems. Rather the experiments recounted below are designed to empirically investigate the usefulness of these concepts 
in some illustrative domains. In Section 2 we present general background on COINs and the experiments we conducted. In Section 3, we present the portion of the COIN framework investigated in this paper. In Sections 4 and 5, we describe the bar and leader-follower experiments, respectively, and how our approach deals with the many pitfalls encountered in each.

\section{MOTIVATION AND BACKGROUND}

A naturally occurring example of a system that can be viewed as COIN is a human economy. For example, one can take the agents to be the individuals trying to maximize their personal rewards. One might then declare that the world utility is a time average of the gross domestic product. ("World utility" per se is not a construction internal to a human economy, but rather something defined from the outside.) To achieve high global utility it is necessary to avoid having the agents work at cross-purposes lest phenomena like the Tragedy of the Commons (TOC) occur, in which individual avarice works to lower global utility [12]. One way to avoid such phenomena is by modifying the agents' utility functions via punitive legislation. A real world example of an attempt to make such a modification was the cration of anti-trust regulations designed to prevent monopolistic practices.

In designing a COIN we have more freedom than anti-trust regulators though, in that there is no base-line "organic" local utility function over which we must superimpose legislation-like incentives. Rather, the entire "psychology" of the individual agents is at our disposal when designing a COIN. This freedom is a major strength of the COIN approach, in that it obviates the need for honesty-elicitation mechanisms, like auctions, which form a central component of conventional economics.

We recently investigated the use of the COIN approach for distributed control of network packet routing [22]. Conventional approaches to packet routing have each router run a shortest path algorithm (SPA), i.e., each router routes its packets in the way that it expects will get those packets to their destinations most quickly. Unlike with a COIN, with SPA-based routing the routers have no concern for the possible deleterious side-effects of their routing decisions on the global goal (e.g., they have no concern for whether they induce bottlenecks). We ran simulations that demonstrated that a COIN-based routing system has better throughputs than does an SPA-based system [22].

In this paper we present a more fine-grained investigation of our COIN methodology, in which we disentangle two of the major components of that methodology and examine the real-world usefulness of them separately. In the first set of experiments we investigate how one might initialize a COIN (i.e., initialize each agents' local reward function) to ensure that the agents do not work at cross purposes. The problem we chose for this purpose is a more challenging variant of Arthur's bar attendance problem [1, 7, 17]. In this problem, agents have to determine which night in the week to attend a bar. The problem is set up so that if either too few people attend (boring evening) or too many people attend (crowded evening), the total enjoyment of the attendees drops. Our goal is to design the reward functions of the attendees so that the total enjoyment across all nights is maximized. (This problem is similar to a number of problems arising in electronic commerce, where agents need to select the "best" markets to trade their wares.)

In the second set of experiments we investigate how run-time modification of agent utility functions and/or inter-agent interactions can improve performance beyond that of the initialized COIN. For this set of experiments we chose a problem where certain "follower" agents mimic the activities of other "leader" agents. The idea is to investigate a scenario where actions that are beneficial to a particular agent have deleterious global effects when copied by other agents.

\section{COIN FRAMEWORK}

As applied here, the COIN framework has three main components:

i. The first component investigates various formalizations of the desideratum that whenever the local utility functions increase, then so must the value of the world utility. It implicitly defines equivalence classes of local utility functions that meet those formalizations, for allowed agent interactions of various types.

ii. There is little explicit concern for the dynamical nature of the COIN in this first component of the framework; that dynamics is subsumed under the assumption that the agents can achieve large values of their local utility functions, via their RL learning. However due to that dynamics it may be that the agents are all inadvertently "frustrating" each other, so that none of them can achieve large values of their local utility functions. The second component of the COIN framework addresses this problem by means of further restrictions (beyond those of the first component) on the allowed set of local utility functions. We call the design of a system's local utility functions using the two components of the COIN framework the COIN initialization of that system.

iii. Successful COIN initialization ensures that if the agents in the system achieve large values of their local utility functions, then the world utility is also large. However due to uncertainties in the type of the agent interactions, in the real world the local utility functions set in the COIN initialization are usually only a good first guess. The third component of the COIN framework addresses this issue by modifying the local utility functions at run-time based on localized statistical information. We call this modifying process macrolearning. In contrast, we call the reinforcement learning that agents perform to optimize their local reward $\mathrm{mi}$ crolearning.

In this paper we consider the state of the system across a set of discrete, consecutive time steps, $t \in\{0,1, \ldots\}$. Without loss of generality, we let all relevant characteristics of an agent at time $t$ including its internal parameters at that time as well as its externally visible actions - be encapsulated by a Euclidean vector $\underline{\zeta}_{\eta, t}$. We call this the "state" of agent $\eta$ at time $t$, and let $\underline{\zeta}$ be the state of all agents across all time. World utility, $G(\underline{\zeta})$, is a function of the state of all agents across all time. Our goal as COIN designers is to maximize world utility.

The following concepts and definitions form the core of the COIN framework:

Subworlds: Subworlds are the sets making up an exhaustive partition of all agents. For each subworld, $\omega$, all agents in that subworld have the same subworld utility function $g_{\omega}(\zeta)$ as their local utility functions. Accordingly, consider having each subworld be a set of agents that collectively have the most effect on each other. In this situation, by and large, agents cannot work at cross-purposes, since all agents that affect each other substantially share the same local utility.

Constraint-alignment: Associated with subworlds is the concept of a (perfectly) constraint-aligned system. That is a system in which any change to the state of the agents in subworld $\omega$ at time 0 will have no effect on the states of agents outside of $\omega$ at times later than 0 . Intuitively, a system is constraint-aligned if no two agents in separate subworlds affect each other, so that the rationale behind the use of subworlds holds. (In the real world of course, systems 
are rarely exactly constraint-aligned.)

Subworld-factored: A subworld-factored system is one where for each subworld $\omega$ considered by itself, a change at time 0 to the states of the agents in that subworld results in an increased value for $g_{\omega}(\zeta)$ if and only if it results in an increased value for $G(\zeta)$. For a subworld-factored system, the side effects on the rest of the system of $\omega$ 's increasing its own utility do not end up decreasing world utility. For these systems, the separate agents successfully pursuing their separate goals do not frustrate each other as far as world utility is concerned.

The definition of subworld-factored is carefully crafted. In particular, it does not concern changes in the value of the utility of subworlds other than the one changing its state. It also does not concern changes to the states of agents in more than one subworld at once. Indeed consider the following property: any change at time 0 to the entire system that improves all subworld utilities simultaneously also improves world utility. This might seem an appealing alternative desideratum to subworld-factoredness. However one can construct examples of systems that obey this property and yet quickly evolve to a minimum of world utility (for example this is the case in the TOC).

It can be proven that for a subworld-factored system, when each of the agents' reinforcement learning algorithms are performing as well as they can, given each others' behavior, world utility is at a critical point. Correct global behavior corresponds to learners reaching a Nash equilibrium [21]. There can be no tragedy of the commons for a subworld-factored system.

Wonderful life utility: Let $\mathrm{CL}_{\omega}(\zeta)$ be defined as the vector $\underline{\zeta} \bmod -$ ified by clamping the states of all agents in subworld $\omega$, across all time, to an arbitrary fixed value, here taken to be 0 . The wonderful life subworld utility (WL) is:

$$
g_{\omega}(\underline{\zeta}) \equiv G(\underline{\zeta})-G\left(\mathrm{CL}_{\omega}(\underline{\zeta})\right) .
$$

When the system is constraint-aligned, so that, loosely speaking, subworld $\omega$ 's "absence" would not affect the rest of the system, we can view the WL utility as analogous to the change in world utility that would have arisen if subworld $\omega$ "had never existed". (Hence the name of this utility - cf. the Frank Capra movie.) Note however, that $\mathrm{CL}$ is a purely mathematical operation. Indeed, no assumption is even being made that $\mathrm{CL}_{\omega}(\zeta)$ is consistent with the dynamics of the system. The succession of states the agents in $\omega$ are clamped to in the definition of the WL utility need not obey the dynamical laws of the system.

This dynamics-independence is a crucial strength of the WL utility. It means that to evaluate the WL utility we do not try to infer how the system would have evolved if all agents in $\omega$ were set to 0 at time 0 and the system evolved from there. So long as we know $\zeta$ extending over all time, and so long as we know $G$, we know the value of WL utility. This is true even if we know nothing of the dynamics of the system.

Another crucial advantage of the WL utility arises from the fact that in a COIN, each agent, by itself, is operating in a large system of other agents, and therefore may experience difficulty discerning the effects of its actions on its utility. Very often this problem is obviated by using the WL utility; the subtraction of the clamped term in the WL utility removes some of the "noise" of the activity of agents in other subworlds, leaving only the underlying "signal" of how the agents in the subworld at hand affect the utility. This makes it easier for the microlearning to improve the utility of the agents in that subworld. When the system is subworld factored, this property facilitates the improvement of world utility. In many circumstances, this cancelling characteristic of WL also ensures that the utility functions obey the applicable a priori locality restrictions on communications among agents.
The experiments in this paper revolve around the following fact: a constraint-aligned system with wonderful life subworld utilities is subworld-factored. Combining this with our subworld-factored systems have Nash equilibria at previous result that subworld-factored systems are at equilibrium at critical points of world utility, this result leads us to expect that a constraint-aligned system using WL utilities in the microlearning will approach near-optimal values of the world utility.

No such assurances accrue to WL utilities if the system is not constraint-aligned however. Accordingly our first set of experiments investigates how well a particular system performs when WL utilities are used but little attention is paid to ensuring that the system is constraint-aligned. Our second set of experiments then focus on a macrolearning algorithm that modifies subworld memberships dynamically, so as to increase the degree of constraint-alignment, all while using WL local utility functions.

\section{BAR PROBLEM}

\subsection{Experimental Method}

We conducted two sets of experiments to investigate the COIN framework. The first set is a variant of Brian Arthur's bar-attendance model [1]. Since we are not interested here in directly comparing our results to those in $[1,13]$, we use a more conventional (and arguably "dumber") reinforcement learning algorithm than the ones investigated in $[1,13]$.

There are $N$ agents, each of whom picks one of seven nights to attend a bar the following week, a process that is then repeated. In each week, each agent uses its own reinforcement learning (RL) algorithm to decide which night to attend to maximize its utility.

The world utility is of the form:

$$
G(\underline{\zeta})=\sum_{t} \sum_{k=1}^{7} \gamma_{k}\left(x_{k}(\underline{\zeta}, t)\right)
$$

where " $x_{j}(\underline{\zeta}, t)$ " means the $j$ 'th component of $x(\underline{\zeta}, t)$ which is the attendance on night $j$ at week $t ; \gamma_{k}(y) \equiv \alpha_{k} y \exp (-y / c) ; c$ and each of the $\left\{\alpha_{k}\right\}$ are real-valued parameters. Intuitively, this world utility is the sum of the world "rewards" for each night in each week.

This $G$ is chosen to reflect the effects in the bar as the attendance profile of agents changes. In the case when there are too few agents, the bar suffers from lack of activity and therefore the world utility should be low. Conversely, when there are too many agents the bar becomes overcrowded and the rewards should also be low. Note that $\gamma_{k}(\cdot)$ reaches its maximum when its argument equals $c$.

The $\alpha$ 's are used to weight the importance of different nights. Two different choices of the $\left\{\alpha_{k}\right\}$ are investigated. One treated attendance on all of the nights equally, $\alpha=\left[\begin{array}{lllllll}1 & 1 & 1 & 1 & 1 & 1 & 1\end{array}\right]$. The other is only concerned with the attendance on one night of the week, $\alpha=\left[\begin{array}{lllllll}0 & 0 & 0 & 7 & 0 & 0 & 0\end{array}\right]$. In our experiments, $c$ was 6 and $N$ is chosen to be significantly larger (4 times) than the number of agents necessary to have $c$ agents attend the bar on each of the seven nights, resulting in 168 agents.

As explicated below, our microlearning algorithms worked by providing a real-valued "reward" signal to each agent at each week $t$. Each agent's reward function is a surrogate for an associated utility function for that agent. The difference between the two functions is that the reward function only reflects the state of the system at one moment in time (and therefore is potentially observable), whereas the utility function reflects the agent's ultimate goal, and therefore can depend on the full history of that agent across time.

We investigated the following functions specifying the rewards at time $t$ for each of the agents: 

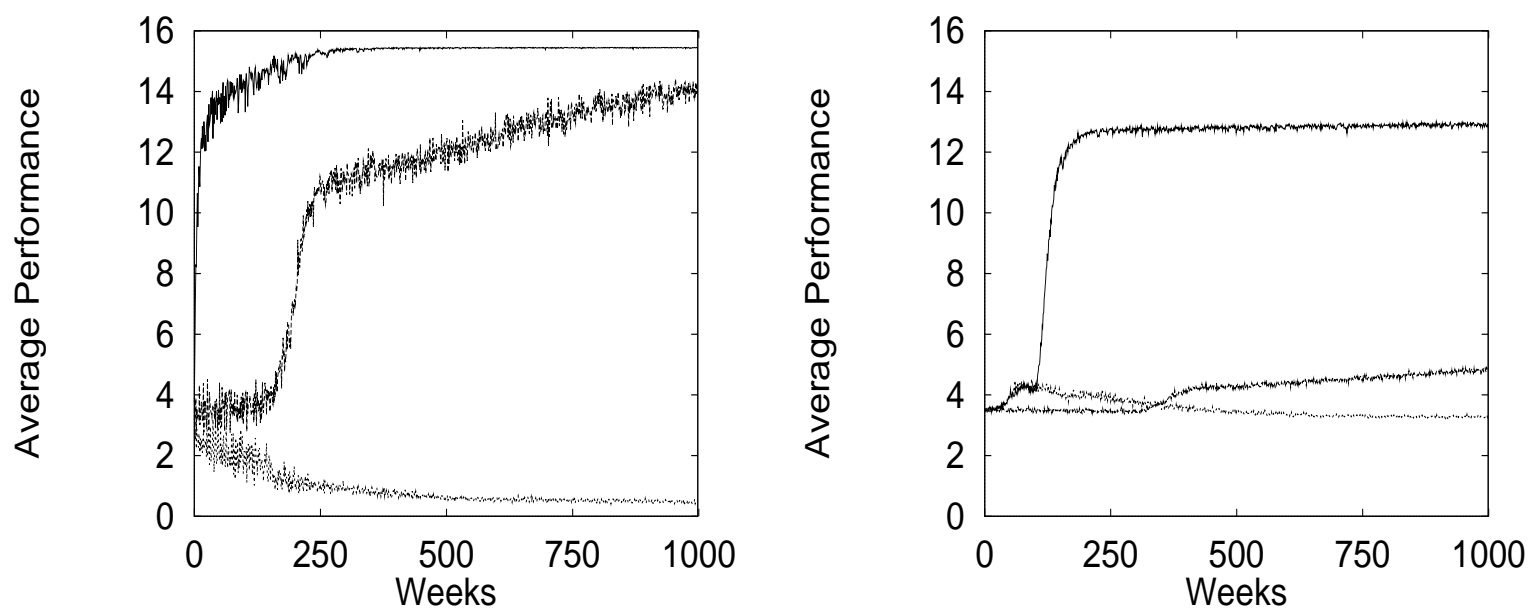

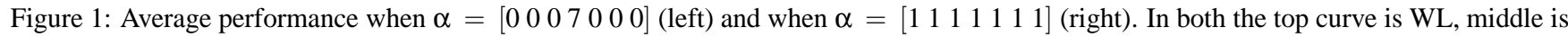
$\mathrm{GR}$, and bottom is UD.

- Uniform Division Reward:

$$
U D\left(d_{\omega}(t), \underline{\zeta}, t\right) \equiv \gamma_{d_{\omega}}\left(x_{d_{\omega}}(\underline{\zeta}, t)\right) / x_{d_{\omega}}(\underline{\zeta}, t)
$$

- Global Reward:

$$
G R\left(d_{\omega}(t), \underline{\zeta}, t\right) \equiv \sum_{k=1}^{7} \gamma_{k}\left(x_{k}(\underline{\zeta}, t)\right)
$$

- Wonderful Life Reward:

$$
\begin{aligned}
& W L\left(d_{\omega}(t), \underline{\zeta}, t\right) \equiv \\
& \quad \sum_{k=1}^{7} \gamma_{k}\left(x_{k}(\underline{\zeta}, t)\right)-\sum_{k=1}^{7} \gamma_{k}\left(x_{k}\left(\mathrm{CL}_{\omega}(\underline{\zeta}), t\right)\right)= \\
& \gamma_{d_{\omega}}\left(x_{d_{\omega}}(\underline{\zeta}, t)\right)-\gamma_{d_{\omega}}\left(x_{d_{\omega}}\left(\mathrm{CL}_{\omega}(\underline{\zeta}), t\right)\right)
\end{aligned}
$$

where $d_{\omega}$ is the night selected by subworld $\omega$. Note the distinction between utilities and rewards. For example, world utility is the sum over all time of global reward.

The UD reward is considered a natural "naive" choice of an agent's reward function; the total reward on each night gets uniformly divided among the agents attending that night.

Providing the GR reward at time $t$ to each agent is considered a reasonable way to provide that agent an approximation to the value of world utility that would ensue if the current policies of all the agents were henceforth frozen to their current polices. This reward function results in all agents receiving the same feedback information. For this reward, the system is automatically subworldfactored. However, evaluation of this function requires centralized communication.

Similarly, the WL reward at time $t$ is considered a reasonable approximation to the WL utility. However, in contrast to the GR reward, to evaluate its WL reward each agent only needs to know the total attendance on the night it attended. In addition, as indicated above, one would expect that with the WL reward the microlearners can readily discern the effects of their rewards on their utilities - something not necessarily true if they use the GR reward.

Each agent is its own subworld. Each agent's microlearning algorithm uses a seven dimensional Euclidean vector to represent the expected reward for each night of the week. At the end of each week, the component of this vector corresponding to the night attended is proportionally adjusted towards the actual subworld reward just received. At the beginning of each week, the agent picks the night to attend using a Boltzmann distribution with energies given by the components of the vector of expected rewards. A decaying temperature is used to aid exploration in the early stage of the process. The microlearning algorithms are set to use the same parameters (i.e. learning rate, Boltzmann temperature, decay rates) for all three reward functions. This learning algorithm is equivalent to Claus and Boutilier's [8] independent learner algorithm for multi-agent reinforcement learning.

\subsection{Results}

Figure 1 presents world reward values as a function of time for the bar problem, averaged over 50 separate runs. Note that world utility is the sum over time of world reward. We present the performance for all three subworld reward functions for both $\alpha=\left[\begin{array}{lllllll}1 & 1 & 1 & 1 & 1 & 1 & 1\end{array}\right]$

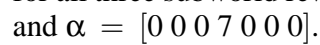

Systems using the WL reward converged to optimal performance. This indicates that the bar problem is sufficiently constraintaligned so that interactions between subworlds does not diminish performance. Presumably this is true because the only interactions between subworlds occurred indirectly, via the microlearning.

Moreover, since the WL reward is easier for the microlearners to exploit (see above), one would expect the convergence using the WL reward to be far quicker than that using the GR reward. The GR reward does eventually converge to the global optimum. This is in agreement with the results obtained by Crites [9] for the bank of elevators control problem. However, when $\alpha=\left[\begin{array}{lllllll}0 & 0 & 0 & 7 & 0 & 0 & 0\end{array}\right]$ the GR reward converged in 1250 weeks. This is more than 4 times the convergence time for the WL reward. When $\alpha=\left[\begin{array}{lllllll}1 & 1 & 1 & 1 & 1 & 1 & 1\end{array}\right]$ the GR reward took 6500 weeks to converge, which was more than 30 times the time WL reward took to converge. This slow convergence is a result of the reward signal being diluted by the large number of agents in the system.

In contrast to the behavior for reward functions based on the COIN framework, use of the UD subworld reward function results in very poor world reward values that deteriorated as the microlearning progressed. For the case where world reward only depends on a single night, this is essentially an instance of the tragedy of the commons; it is in every agent's interest to attend the same night, and their doing this shrinks the world reward "pie" that must be divided among all agents.

Taken together, these experiments demonstrate that the initialization prescriptions of the COIN framework can result in excellent global performance. 


\section{LEADER-FOLLOWER PROBLEM}

\subsection{Experimental Method}

In the experiments recounted in the previous section the system was sufficiently constraint-aligned for the WL reward to result in optimal performance. The second set of experiments involving leaders and followers also used WL reward. These experiments investigated the use of macrolearning to make an initially non-aligned system more constraint-aligned, and thereby improve the world utility. In this experiment, macrolearning consists of changing the subworld memberships of the agents.

In these experiments the bar problem is modified to incorporate constraints designed to frustrate WL subworld reward. This is done by forcing the nights picked by some agents (followers) to agree with those picked by other agents (leaders). Each leader has two followers. The world utility is the sum, over all leaders, of the values of a triply-indexed reward matrix whose indices are the the nights that the leader and his two followers attend:

$$
G(\underline{\zeta})=\sum_{t} \sum_{i} R_{l_{i}(t), f 1_{i}(t), f 2_{i}(t)}
$$

where $l_{i}(t)$ is the night the $i^{t h}$ leader attends, and $f 1_{i}(t)$ and $f 2_{i}(t)$ are the nights attended by the followers of leader $i$, in week $t$. The system's dynamics is what restricts all the members of each triple $\left(l_{i}(t), f 1_{i}(t), f 2_{i}(t)\right)$ to equal the night picked by leader $i$ for week $t$. However, $G$ and $R$ are defined for all possible triples, $l_{i}(t), f 1_{i}(t)$ and $f 2_{i}(t)$. So in particular, $R$ is defined for dynamically unrealizable triples that arise in the clamping operation. Because of this, for certain $R$ 's there exists subworld memberships such that the dynamics assures poor world utility when WL rewards are used. This is precisely the type of problem that macrolearning is designed to correct.

To investigate the efficacy of the macrolearning, two sets of separate experiments were conducted. In the first one the reward matrix $R$ is chosen so that when leader and follower agents are placed in separate subworlds, leaders maximizing their WL reward results in minimal world reward. In contrast to the bar problem experiments, in these experiments, due to the coupling between leaders and followers, having each agent be its own subworld would mean badly violating constraint-alignment. (The idea is that by changing subworld memberships macrolearning can correct this and thereby induce constraint-alignment.) In the second set of experiments, rather than hand-crafting the worst-case reward matrix, we investigate the efficacy of macrolearning for a broader spectrum of reward matrices generated randomly.

For the worst-case reward matrix, the subworld membership that results in the most constraint-aligned system is when a leader and associated followers belong to the same subworld. The least constraint-alignment occurs when the leader and associated followers are all in separate subworlds. In addition to these two cases we also investigate random subworld membership. We investigate all these cases both with and without macrolearning, and with both worst case and a full spectrum of random reward matrices. The microlearning in these experiments is the same as in the bar problem. All leader-follower experiments use the WL subworld reward.

When macrolearning is used, it is implemented after the microlearning has run for a specified number of weeks. The macrolearning works by estimating the correlations between the nights picked by the agents. This is done by examining the attendances of the agents over the preceding weeks. The agents estimated to be the most correlated with one another are grouped into the same subworld, with the restriction that the number of agents per subworld is always three.

\subsection{Results}

Figure 2 presents performance averaged over 50 runs for world reward as a function of weeks using the worst case reward matrix. For comparison purposes, the top curve represents the case where the leaders and their followers are placed in the same subworld so that there is perfect constraint-alignment. The bottom curve represents the other extreme where the leaders and their followers are placed in different subworlds resulting in minimal constraint-alignment. In both plots, the middle curve shows the performance of randomly initialized subworlds with (right) and without (left) macrolearning.

The performance for randomly assigned subworlds differs only slightly from that of having each agent be its own subworld; both start with poor values of world reward that deteriorates with time. However, when macrolearning is performed on systems with initially random subworld assignments, the system quickly rectifies itself and converges to optimal performance. This is reflected by the sudden vertical jump through the middle of the right plot at 500 weeks, the point at which macrolearning changes the subworld memberships. This demonstrates that by changing the subworld memberships macrolearning results in perfect constraint-alignment, so that the WL subworld reward function quickly induces the maximal value of the world reward.

Figure 3 presents performance averaged over 50 runs for world reward as a function of weeks using a spectrum of reward matrices selected at random. The ordering of the plots is exactly as in Figure 2. Macrolearning is applied at 2000 weeks, in the right plot. The simulations in Figure 3 were lengthened from those in Figure 2 because the convergence time are longer.

The macrolearning algorithm results in a transient degradation in performance at 2000 weeks followed by convergence to the optimal. Without macrolearning the system's performance no longer varies after 2000 weeks. Combined with the results presented in Figure 2, these experiments demonstrate that the COIN construction of macrolearning works quite well.

\section{CONCLUSION}

For MAS's to fulfill their full potential, even when used in large systems having strong limitations on inter-agent interactions and communication, a way is needed to automatically configure/update the system to achieve the provided global goal. One approach to this problem is to have each agent run a reinforcement learning algorithm and then configure/update the associated reward functions (and other characteristics) of the agents so as to facilitate achievement of the global goal. This is the central concept embodied in COINs.

In this paper we presented a summary of (a portion of) the mathematical framework of COINs. We then presented two sets of experiments empirically validating the predictions of that framework. The first set of experiments considered difficult variants of Arthur's famous El Farol Bar problem. These experiments showed that even when the conditions required by the theorems of how to initialize a COIN do not hold exactly, they often hold well enough so that they can be applied with confidence. In those experiments, the COINs quickly achieved optimal performance despite the local nature of the information available to each agent, and in particular the COINs automatically avoided the tragedy of the commons that was designed into those experiments. This was not true when the reward functions were either set in a "naive" manner or were all set to equal the global reward.

The second set of experiments considered leader-follower problems that were hand-designed to cause maximal difficulty for those COIN initialization theorems. These experiments explicitly tested the run-time updating procedures of the COIN framework for overcoming such initialization problems. Here, as expected, the initial 

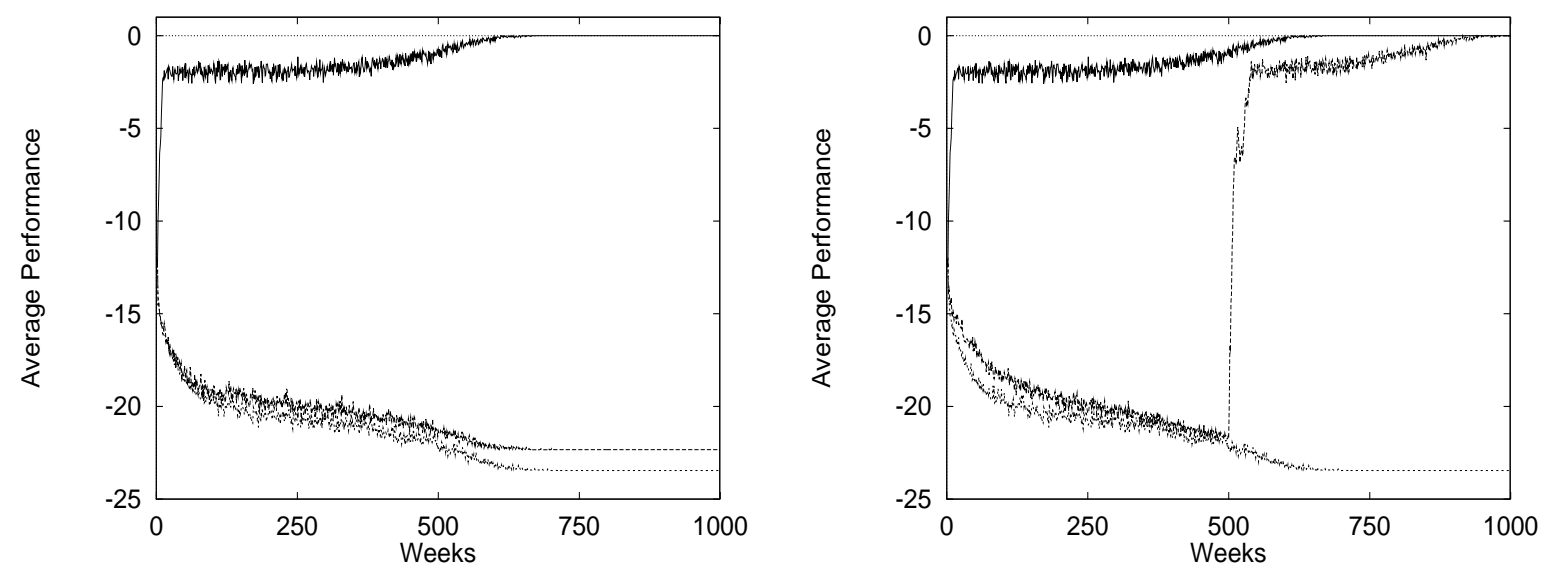

Figure 2: Leader-follower problem with worst case reward matrix. In both plots the top curve represents perfect constraint-alignment, the bottom curve represents minimal constraint-alignment, and the middle curves represent random subworlds without (left) and with (right) macrolearning at 500 weeks.
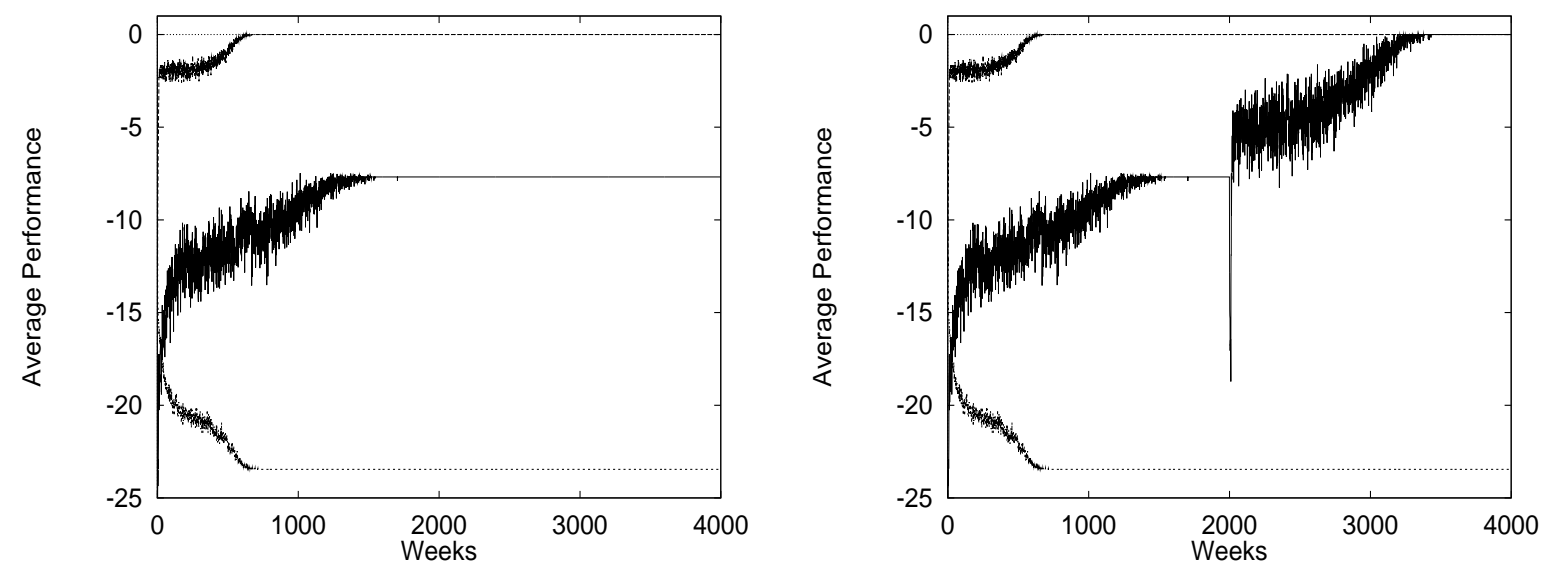

Figure 3: Leader-follower problem for full spectrum reward matrices. The ordering of the plots is exactly as in Figure 3. Macrolearning is applied at 2000 weeks, in the right plot.

performance of the COIN was quite poor. However once the updating procedures were brought online, performance quickly rose to optimal. Again, this was in contrast to the case where the reward functions were either set in a "naive" manner or were all set to equal the global reward.

The conclusion of these experiments is that the prescriptions of the COIN framework for how to configure a large MAS often apply even when the exact conditions required by the associated theorems do not hold. Moreover, in those relatively unusual circumstances when the initialization prescriptions of the COIN theorems do not result in optimal global performance, the run-time updating component of the framework can rectify the situation, so that optimal performance is achieved.

Acknowledgments: The authors would like to thank Ann Bell, Hal Duncan and Jeremy Frank for helpful discussions.

\section{References}

[1] W. B. Arthur. Complexity in economic theory: Inductive reasoning and bounded rationality. The American Economic Review, 84(2):406-411, May 1994.

[2] E. Baum. Manifesto for an evolutionary economics of intelli- gence. In C. M. Bishop, editor, Neural Networks and Machine Learning. Springer-Verlag, 1998.

[3] J. Berg and A. Engel. Matrix games, mixed strategies, and statistical mechanics. preprint cond-mat/9809265, 1998.

[4] C. Boutilier, Y. Shoham, and M. P. Wellman. Editorial: Economic principles of multi-agent systems. Artificial Intelligence Journal, 94:1-6, 1997.

[5] J. M. Bradshaw, editor. Software Agents. MIT Press, 1997.

[6] G. Caldarelli, M. Marsili, and Y. C. Zhang. A prototype model of stock exchange. Europhys. Letters, 40:479-484, 1997.

[7] D. Challet and Y. C. Zhang. Emergence of cooperation and organization in an evolutionary game. Physica A, 246(3-4):407, 1997.

[8] C. Claus and C. Boutilier. The dynamics of reinforcement learning cooperative multiagent systems. In Proceedings of the Fifteenth National Conference on Artificial Intelligence, pages 746-752, June 1998.

[9] R. H. Crites and A. G. Barto. Improving elevator performance using reinforcement learning. In D. S. Touretzky, M. C. 
Mozer, and M. E. Hasselmo, editors, Advances in Neural Information Processing Systems - 8, pages 1017-1023. MIT Press, 1996.

[10] D. Fudenberg and D. K. Levine. The Theory of Learning in Games. MIT Press, Cambridge, MA, 1998.

[11] D. Fudenberg and J. Tirole. Game Theory. MIT Press, Cambridge, MA, 1991.

[12] G. Hardin. The tragedy of the commons. Science, 162:12431248, 1968.

[13] J. Hu and M. P. Wellman. Multiagent reinforcement learning: Theoretical framework and an algorithm. In Proceedings of the Fifteenth International Conference on Machine Learning, pages 242-250, June 1998.

[14] J. Hu and M. P. Wellman. Online learning about other agents in a dynamic multiagent system. In Proceedings of the Second International Conference on Autonomous Agents, pages 239246, May 1998.

[15] B. A. Hubermann and T. Hogg. The behavior of computational ecologies. In The Ecology of Computation, pages 77115. North-Holland, 1988.

[16] N. R. Jennigs, K. Sycara, and M. Wooldridge. A roadmap of agent research and development. Autonomous Agents and Multi-Agent Systems, 1:7-38, 1998.

[17] N. F. Johnson, S. Jarvis, R. Jonson, P. Cheung, Y. R. Kwong, and P. M. Hui. Volatility and agent adaptability in a selforganizing market. preprint cond-mat/9802177, February 1998.

[18] T. Sandholm, K. Larson, M. Anderson, O. Shehory, and F. Tohme. Anytime coalition structure generation with worst case guarantees. In Proceedings of the Fifteenth National Conference on Artificial Intelligence, pages 46-53, 1998.

[19] S. Sen. Multi-Agent Learning: Papers from the 1997 AAAI Workshop (Technical Report WS-97-03. AAAI Press, Menlo Park, CA, 1997.

[20] K. Sycara. Multiagent systems. AI Magazine, 19(2):79-92, 1998.

[21] D. Wolpert and K. Tumer. An overview of collective intelligence. In J. M. Bradshaw, editor, Handbook of Agent Technology. AAAI Press/MIT Press, 1999.

[22] D. Wolpert, K. Tumer, and J. Frank. Using collective intelligence to route internet traffic. In Advances in Neural Information Processing Systems - 11. MIT Press, 1999.

[23] D. Wolpert, K. Wheeler, and K. Tumer. Collective intelligence for distributed control. 1999. (pre-print). 